\title{
Extubation of the Challenging or Difficult Airway
}

\author{
Matteo Parotto ${ }^{1,2,3}$ (1) $\cdot$ Richard M. Cooper ${ }^{1}$ Elizabeth C. Behringer ${ }^{4}$ \\ Published online: 4 September 2020 \\ (C) Springer Science+Business Media, LLC, part of Springer Nature 2020
}

\begin{abstract}
Purpose of Review This review explores relevant definitions, epidemiology, management, and potential future research directions in the extubation of the challenging/difficult airway. It provides guidance on identifying patients at risk and how to approach these clinical scenarios.

Recent Findings Based on recent literature, including large-scale audits and closed claims analysis, it is increasingly recognized that extubation of the difficult airway is a situation at risk of severe adverse events. Some strategies to manage the extubation of the challenging/difficult airway have been described.

Summary Extubating the challenging/difficult airway is a high-risk situation. However, it is fundamental to keep in mind that intended extubation is always an elective procedure. As such, it is imperative to adhere to principles of careful patient and context assessment, planning, and execution only when optimal conditions have been secured.
\end{abstract}

Keywords Endotracheal intubation · Extubation · Airway management $\cdot$ Airway exchange catheter $\cdot$ Supraglottic airway

\section{Introduction}

Extubation is an essential procedure in anesthesia, when endotracheal intubation is required, as well as in critical care medicine, when mechanical ventilatory support is needed. While safely performed in a large proportion of cases, it can present significant challenges and complications. Complications associated with extubation can be severe,

Literature search strategy: a search of MEDLINE, CINAHL, Embase, Web of Science, and CENTRAL for published English-language relevant papers was conducted. The search was run periodically, last on May 23, 2020. The terms included "extubation," "endotracheal extubation," "difficult extubation," and "challenging extubation." The references of retrieved and selected articles were reviewed to identify additional potentially relevant sources.

This article is part of the Topical Collection on Airway Management

Matteo Parotto

matteo.parotto@uhn.ca

Richard M. Cooper

richard.cooper@utoronto.ca

Elizabeth C. Behringer

DrB02@yahoo.com; Elizabeth.C.Behringer@kp.org

1 Department of Anesthesiology and Pain Medicine, University of Toronto, 12th Floor, 123 Edward Street, Toronto, ON M5G 1E2, Canada resulting in major morbidity and mortality [1]. In this review, we explore relevant definitions, epidemiology, and management of extubation as well as future research opportunities. We will not focus on the management of low-risk extubations; however, many of the principles outlined here may apply to those circumstances as well.

Of note, extubation is considered an aerosol-generating medical procedure (AGMP) [2], and according to the specific
2 Department of Anesthesia and Pain Management, Toronto General Hospital, EN 429 - 200 Elizabeth Street, Toronto, ON M5G 2C4, Canada

3 Interdepartmental Division of Critical Care Medicine, University of Toronto, Toronto, ON, Canada

4 Division of CardioVascular Surgery and Critical Care, Kaiser Permanente Los Angeles Medical Center, 1526 N Edgemont, Los Angeles, CA 90027, USA 
circumstances, appropriate personal protective equipment (PPE) should be adopted to ensure the safety of all personnel involved. This topic is discussed elsewhere [2].

\section{Definitions}

Extubation may be challenging due to the risk of failure. Extubation failure occurs when the patient cannot maintain oxygenation, alveolar ventilation, airway patency, pulmonary toilet and secretion management, airway protection, or any combination of these issues. Extubation may be challenging due to the predicted difficulty with reintubation should this be required. Reintubation may be difficult when pre-existing difficulty with airway management exists and changes to the airway have occurred due to trauma, positioning, or prolonged mechanical ventilation. Additionally, other factors may play an important role in determining difficulty, such as the location where reintubation occurs, the availability of expert help and essential team members, and patient's clinical conditions such as rapid desaturation and hypotension [3•]. Although this chapter focuses mainly on intended extubation, there are circumstances in which extubation is unplanned. Unplanned extubation is defined as unintentional and uncontrolled removal of the endotracheal tube due to either actions of the patients or during nursing care or movement of the patient (e.g., during prone positioning) [4]. It occurs more frequently in critical care, pre-hospital, and emergency medicine and is associated with significant morbidity and mortality [3•].

Complications of extubation may be either minor or major. Minor complications may be transient and not require reintubation. They are frequently not reported. Minor complications include transient hypertension, tachycardia, coughing, bucking, and agitation. However, these physiological derangements may result in potentially serious complications in certain patient populations. Patients with significant cardiac or cerebrovascular disease and pre-existing elevated intracranial or intraocular pressure and patients who have undergone neck surgery, neurosurgery, and some plastic surgical procedures are at risk for the development of serious sequelae.

Major complications usually refer to extubation failure, which is generally defined as the need for reintubation within 24-72 $\mathrm{h}$ after extubation [1], although a clear consensus on a precise definition or time frame does not exist at present [3•].

Extubation failure due to airway-related problems (e.g., upper airway obstruction) occurs due to the inability to breathe without a tracheal tube [5]. Other complications at the time of extubation include pulmonary aspiration (in fact, it is reported that one-third of cases of pulmonary aspiration occur after extubation) and hypoxemia due to causes different than upper airway obstruction (e.g., post-operative pulmonary complications such as atelectasis, or respiratory depression due to medication effects). Typically, both in anesthesia and critical care settings, extubation failure frequently occurs within $2 \mathrm{~h}$ of extubation, and largely in the first $24 \mathrm{~h}$ following extubation $[1,6]$. Extubation failure and difficult or failed reintubation may result in life-threatening complications such as prolonged hypoxia and cardiac arrest, with consequent brain damage and/or death. Unplanned extubation is associated with significant complications, including aspiration pneumonia, hypoxemia, arrhythmias, vocal cord injury, brain damage, and death.

\section{Epidemiology}

The rates of extubation failure among adult patients in the operating room (OR) and post-anesthesia care unit (PACU) are between 0.1 and $0.45 \%$ [1]. Unplanned extubation incidence may be higher. Data from the literature range widely, with reported rates between 0.3 and $35.8 \%$ in intensive care units [4]. The incidence in the operating room is unknown and may be underreported. Reasons for early reintubation include respiratory insufficiency, airway obstruction, bronchospasm, inadequate reversal of neuromuscular blockade, and druginduced respiratory depression [1]. In the intensive care unit (ICU), rates of extubation failure are $2-25 \%$ and are generally due to airway obstruction, excess secretions, inability to protect the airway, and respiratory failure [7]. The 4th National Audit Project (NAP4) in the UK found that major airway complications following extubation were responsible for approximately one-third of the reported cases related to anesthesia, and all reported complications during emergence or recovery were due to airway obstruction [8]. There were 38 reported cases related to extubation failure; hypoxia due to airway obstruction resulted in cardiac arrest in five patients, two of whom died. Prolonged hypoxia occurred in approximately one-half of the 38 reported cases, and a surgical airway was attempted in 10 patients. In the 2000-2012 American Society of Anesthesiologists closed claims analysis, there were 102 events related to difficult tracheal intubation; a significant proportion of these events occurred after extubation. Approximately $20 \%$ of attempted reintubations following surgery were notable for a significant risk of brain injury or death [9॰].

\section{Management}

The most fundamental principle of intended extubation is that this procedure is always elective. As such, careful assessment and planning must occur, and extubation should not be attempted until optimal conditions have been achieved. In the vast majority of the cases reported in the 2000-2012 closed claimed analysis, there was no back-up plan for emergent reintubation [9•]. 


\section{Risk Factors}

First and foremost, it is crucial to identify patients at risk of extubation failure and its complications, should reintubation be required. These include both patient- and procedure-related factors $[1,3 \cdot, 8,9 \bullet, 10]$.

\section{Patient Factors}

- Laryngospasm

- Obesity

- Obstructive sleep apnea

- Chronic obstructive pulmonary disease (COPD)

- Neuromuscular diseases

- Head and neck radiation history

- Parkinson disease

- Rheumatoid arthritis

- Paradoxical vocal fold motion

- Female sex

- Advanced age ( $>70$ years)

- Anatomically difficult airway. It is important to note that an easy intubation accomplished under controlled elective conditions does not necessarily equate to optimal conditions should an emergent reintubation be required [3•]

- Physiological difficulty. Physiologic abnormalities such as hypoxemia, hypercapnia, acidosis, and cardiovascular instability at the time of emergent reintubation may render patients less tolerant of delays and persistent or prolonged intubation attempts.

\section{Surgical/Procedural Factors}

- Head and neck surgical procedures (Table 1) [1, 3•]. In the NAP4 registry, adverse events at the time of extubation in patients with head and neck pathologies accounted for $34.2 \%$ of all events during emergence or recovery [8]

- Neurologic injuries affecting vocal cord motion, dynamic airway collapse, or airway compression above, below, or at the level of the larynx

- Restricted airway access, e.g., Halo fixation, cervical collar, jaws wired shut

- Prolonged duration of mechanical ventilation

- Airway edema due to positioning, airway trauma during initial airway management or fluid overload

Additionally, we need to evaluate contextual challenges. The emergent nature of a reintubation may result in insufficient information, medication, equipment, qualified personnel, suitable positioning, or access to the airway. Other human
Table 1 Examples of head and neck surgical procedures at high risk of extubation failure and complications should reintubation be required

Upper airway endoscopy

Thyroidectomy

Debulking of large oral cancers

Oral and neck reconstructions requiring surgical flaps

Carotid endarterectomy

Anterior cervical spine repairs especially with a bleeding diathesis

Maxillomandibular fixation

Forced cervical flexion by a "guardian stitch" between the chin and sternum in tracheal resection surgeries

factors such as operator fatigue, situational awareness, stress, and urgency may interfere with an organized, appropriate, and well-executed approach to managing the airway [3•].

Risk factors for unplanned extubation include inadequately securing the endotracheal tube, prone or lateral positioning, position changes, patient transport (for example, ambulance transport or in-hospital transfer to magnetic resonance imaging or computerized tomography scans), delirium, and agitation (in particular with lack of physical restraints where indicated for patient protection) $[3 \cdot, 4]$.

\section{Assessment of Optimization Prior to Extubation}

\section{Contextual Assessment}

Extubation should not be performed until it has been determined that the patient's medical condition is stable, the airway is patent, potential difficulties in the event of reintubation have been identified, and a plan that includes back-up options has been developed and shared among the team members involved. The required expertise and additional expert help should be present at the bedside or their immediate availability should be confirmed. The availability of the necessary equipment should be checked and confirmed.

\section{Clinical Assessment [11]}

- Respiratory: spontaneous tidal volume $>6 \mathrm{ml} / \mathrm{kg}$, vital capacity $>15 \mathrm{ml} / \mathrm{kg}$, respiratory rate $<30$ breaths $/ \mathrm{min}$, maximum inspiratory pressure $<-20 \mathrm{cmH}_{2} \mathrm{O}$, adequate gas exchange [12]

- Cardiovascular: hemodynamic stability, without significant inotropic support or with stable, moderate support

- Adequate reversal of neuromuscular block reversal, which should be monitored with a quantitative neuromuscular monitor [13]

- Risk of airway edema: Cuff leak test-This test has been used to assess the adequacy of airflow around the cuffdeflated tracheal tube and is used for patients who are at 
risk for airway edema (e.g., patients with significantly positive fluid balance, prolonged Trendelenburg or prone positioning, maxillofacial or neck surgery). It can be performed qualitatively or quantitatively. After suctioning the pharynx, the cuff is slowly deflated. In the qualitative assessment, the tracheal tube is progressively occluded. A spontaneously breathing patient should be able to breathe in and out around the tube. To the extent that this cannot be accomplished, it may reflect either insufficient respiratory power or airway swelling. In the quantitative assessment, in a volume-cycled ventilation mode, the difference between the inspired and expired tidal volume is measured. Cuff leak volumes less than $110 \mathrm{ml}$ or less than 12 to $24 \%$ of the delivered tidal volume are thresholds for determining diminished airway patency and risk for postextubation stridor from laryngeal edema. However, presence of a leak does not rule out the possibility of subsequent airway obstruction or extubation failure of a nonobstructive cause. This test has been reported to have sensitivity and specificity of 56 and $92 \%$, respectively [14].

As highlighted also in the American Thoracic Society recommendations, a cuff leak test is suggested in mechanically ventilated adults who meet extubation criteria and are deemed high risk for post-extubation stridor. Risk factors for postextubation stridor include traumatic intubation, intubation for more than 6 days, large endotracheal tube, female sex, and reintubation after unplanned extubation [15•]. In patients with an absent or reduced cuff leak, administration of a corticosteroid at least $4 \mathrm{~h}$ before extubation is advised. A repeat cuff leak test is not required after the administration of systemic steroids $[15 \bullet]$.

If any of the criteria described in the clinical assessment above are not met, extubation should not be performed, and it should be postponed until the medical condition has improved.

\section{Execution of Extubation of the Challenging Airway}

\section{Deep Extubation}

There is very little evidence but considerable opinion regarding deep vs. awake tracheal extubation. Although deep extubation is commonly practiced in infants who are more susceptible to laryngospasm, it is less widely used in adults. The advantages of deep extubation include the potential for earlier transport to the recovery room and reduced airway and hemodynamic stimulation during extubation. Disadvantages include increased risk of aspiration and airway obstruction. Expert opinion advises against deep extubation of the patient with a higher risk airway [16•].

\section{Medications to Reduce Coughing}

Medications may assist in reducing coughing on extubation, including dexmedetomidine, remifentanil, fentanyl, and lidocaine via intracuff, tracheal/topical, and i.v. routes. Dexmedetomidine may be the most effective drug in these regards [17].

Airway Exchange Catheters Different international guidelines (American Society of Anesthesiologists Task Force guidelines [18], the Canadian Airway Focus guidelines [19, 20], and the Difficult Airway Society/Royal College of Anaesthetists [16•]) recommend considering the placement of an airway exchange catheter (AEC) when extubating a patient with a difficult airway. AECs are long, hollow semirigid catheters designed and approved for endotracheal tube (ETT) exchange [3•]. The principle of using AECs in the setting of difficult intubation is to insert them through the ETT at a predefined depth. Distance marks on the AEC allow alignment with those on the ETT, to avoid the tip of the AEC protruding beyond that of the ETT, causing trauma or irritation to the trachea, carina, or bronchi. The AEC is left in place with the tip in the trachea after extubation [11]. If reintubation is necessary, the AEC is used as a stylet to guide reinsertion of an ETT. AECs have a high success rate, particularly when a videolaryngoscope is used to facilitate reintubation over the AEC [21]. First attempt and overall success rates have been shown to be higher and the incidence of complications lower when reintubation was performed with an AEC in situ.

It is important to ensure that an AEC is properly positioned and secured (taped in the midline). This will generally result in tolerance of the device without the need for topical anesthetics or additional sedation. Patients can generally talk, cough, and breathe around the device. Intolerance most often indicates that the catheter has been advanced too deeply or has not been adequately secured in the midline of the mouth [3•]. If the patient is receiving enteral feeds via an oro- or nasogastric tube, these should be held while an AEC remains in situ. An AEC should remain in place until conditions that prompted its use have resolved. A common error is its premature removal. If reintubation is required in a patient known to have an anatomically difficult airway, the additional problems resulting from physiological and/or contextual challenges may significantly increase morbidity. We recommend leaving the device in situ until the airway no longer dictates the need for increased vigilance.

The most commonly used AEC is the Cook AEC (Cook Medical, Bloomington IN), available in different sizes with outer diameters (OD) of $8,11,14$, and $19 \mathrm{Fr}$. These are intended for exchange of ETT with inner diameters (ID) of at least $3,4,5$, and $7 \mathrm{~mm}$ respectively. Although it is widely advocated for maintaining airway access following extubation, the most commonly used commercial product 
(Cook AEC) was licensed for immediate tracheal tube exchange in the UK, Canada, Australia, Europe, the USA, and most of Latin America, Asia, and Africa [3•, 22, 23]. Therefore, leaving the AEC in situ for potential reintubation, though widely practiced, is technically an off-label application.

More recently, Cook Medical released a Staged Extubation set specifically designed for extubation of the difficult airway. The Staged Extubation set is licensed in the UK, Canada, Australia, some of Latin America and Africa, and most of Europe, but not the USA [23]. This set consists of a 0.035-in (outer diameter) flexible wire (145 cm long) that is alleged to cause minimal irritation while in situ, and a 14-Fr AEC with a blunt soft tip. The wire is advanced through the ETT prior to extubation and left in situ once the ETT is removed. Should reintubation be required, the $14-\mathrm{Fr}$ catheter is advanced over the wire, and subsequently the ETT is advanced over the catheter [3॰].

AECs are hollow and allow the delivery of oxygen either via a $15-\mathrm{mm}$ connector (for a resuscitation bag or ventilator circuit) or a luer-lock connection for jet ventilation. A significant risk of barotrauma accompanies such applications [24•] and should be limited only to emergency temporizing measures in situations where oxygen supplementation via face mask has failed, and preparation for reintubation is underway. If insufflating oxygen via the AECs, flow should be limited to a maximum of $2 \mathrm{l} / \mathrm{min}$. Jet ventilation via an AEC should generally be avoided and considered only by very expert providers.

\section{Extubation with a Supraglottic Airway}

Supraglottic airways (SGAs) have been utilized in facilitating extubation under general anesthesia in at-risk patients but are not advised for use in patients with anatomically difficult airways. Rather, it should be regarded as a bridge to successful and uneventful emergence from anesthesia. This is considered an advanced maneuver [18]. This approach has been shown to reduce hemodynamic stress and coughing, which may be desirable to minimize the risk of bleeding and hematoma formation after head and neck surgery, for example. It is also useful in settings where the evaluation of vocal cord function prior to awakening is desirable, as a flexible scope can be inserted via the supraglottic airway to perform such assessment. A commonly adopted technique for extubation with a supraglottic airway involves the insertion of the SGA behind the ETT, prior to the removal of the latter. This approach was described by Dr. PM Bailey and has become known as the "Bailey maneuver" [25].

\section{Elective Surgical Tracheostomy}

In some circumstances, it may be unsafe to remove a tracheal tube even if this is postponed for hours or days. Examples of such situations include extensive intraoral flaps, an obstructed airway, significant swelling (either currently present or predictable in the next few hours as a result of surgical interventions), or bleeding in or around the airway [3॰].

Consideration of the performance of an elective surgical tracheostomy is warranted following evaluation by a multidisciplinary team including anesthesiologists, intensivists, and surgeons. Evaluation must include consideration of patient factors as well as an assessment of the resources and skills required to secure a compromised airway on an emergent basis.

Detailed and helpful algorithms to guide the management of extubation have been developed and are available in the Difficult Airway Society/Royal College of Anaesthetists guidelines [16•].

\section{Post-Extubation}

Strategies to prevent failure after extubation include head-up positioning (unless contraindicated) and administration of supplemental oxygen. Noninvasive ventilation and highflow nasal therapies have been suggested as potential strategies in "at-risk" populations [26, 27]. These include, for example, patients who have received mechanical ventilation for longer than $24 \mathrm{~h}$ and have hypercapnia, chronic obstructive pulmonary disease (COPD), congestive heart failure (CHF), or other serious comorbidities. In such situations, NIV may be applied immediately after extubation to realize the outcome benefits. Similarly, high-flow nasal cannula may reduce the risk of reintubation after extubation in patients receiving mechanical ventilation for more than $24 \mathrm{~h}$ [28]. If stridor occurs, nebulized racemic epinephrine, diuretics, and administration of inhaled $\mathrm{O}_{2}$ /helium mixture may have a role, depending on the underlying etiology [3•]. In such circumstances, close monitoring is mandatory and planning for failure and potential difficult reintubation should be undertaken.

\section{Research}

Extubation accounts for a significant proportion of complications of airway management as highlighted in the NAP4 study and in the ASA closed claims analysis. The literature and existing guidelines have largely focused on intubation and less so on extubation. In recent years, the Difficult Airway Society, the French Society of Anaesthesia and Intensive Care, and the American Association of Respiratory Care have developed specific documents with recommendations for extubation, 
although there is still limited scientific evidence to guide safe practices. While important data have emerged from projects such as the NAP4 study and ASA closed claims analysis, these reports have some limitations and may underestimate the incidence of the problem.

Many questions remain unanswered at present. We think research priorities in the field should include large-scale epidemiological studies to accurately identify the scale of extubation-related complications, the accurate identification of incidence and risk factors for complications at extubation, and studies evaluating optimal techniques for managing specific challenging extubation scenarios and how to implement effective training in relevant techniques for the specialists involved.

\section{Summary and Conclusions}

Extubation is an essential procedure in anesthesia and critical care medicine. Extubation of the challenging or difficult airway is a clinical scenario at risk of severe, life-threatening complications. Adverse events at the time of extubation account for a significant proportion of airway managementrelated serious adverse events. It is fundamental for specialists in these fields to identify the situations at increased risk and to implement appropriate planning to take into account contextual as well as patient-specific factors. While a potentially challenging procedure, intended extubation is always elective and as such, it allows for careful preparation.

Future research will further inform how to precisely identify patients at risk of extubation-related complications, optimal techniques, and learning approaches.

\section{Compliance with Ethical Standards}

Conflict of Interest The authors have no conflicts of interest to declare.

\section{References}

Papers of particular interest, published recently, have been highlighted as:

- Of importance

1. Cavallone LF, Vannucci A. Extubation of the difficult airway and extubation failure. Anesth Analg. 2013;116(2):368-83.

2. Cook TM, El-Boghdadly K, McGuire B, et al. Consensus guidelines for managing the airway in patients with COVID-19: guidelines from the Difficult Airway Society, the Association of Anaesthetists the Intensive Care Society, the Faculty of Intensive Care Medicine and the Royal College of Anaesthetists. Anaesthesia. 2020;75(6):785-99.

3. Cooper RM. Extubation following anesthesia. In: UpToDate, Hagberg CA and Crowley M (Eds), UpToDate 2020. Excellent overview of extubation, regularly updated.
4. Berkow L. Unplanned or accidental extubation in the perioperative environment. Anesthesiology News. 2019;27.

5. Epstein SK, Ciubotaru RL. Independent effects of etiology of failure and time to reintubation on outcome for patients failing extubation. Am J Respir Crit Care Med. 1998;158(2):489-93.

6. Mort TC. Continuous airway access for the difficult extubation: the efficacy of the airway exchange catheter. Anesth Analg. 2007;105(5):1357-62.

7. Rothar RC, Epstein SK. Extubation failure: magnitude of the problem, impact on outcomes, and prevention. Curr Opin Crit Care. 2003;9(1):59-66.

8. Cook TM, Woodall N, Frerk C. Fourth National Audit Project of the Royal College of Anaesthetists and Difficult Airway Society. Major complications of airway management in the United Kingdom. Report and Findings. London 2011. Accessed from: http://www.rcoa.ac.uk/nap4. (Accessed on May 23, 2020)

9. Joffe AM, Aziz MF, Posner KL, Duggan LV, Mincer SL. Domino KB. Management of difficult tracheal intubation: a closed claims analysis. Anesthesiology. 2019;131(4):818-29 A recent analysis of severe complications related to airway management and of the risk factors involved in complications.

10. Jaber S, Quintard H, Cinotti R, Asehnoune K, Arnal JM, Guitton C, et al. Risk factors and outcomes for airway failure versus nonairway failure in the intensive care unit: a multicenter observational study of 1514 extubation procedures. Crit Care. 2018;22(1):236.

11. Artime CA, Hagberg CA. Tracheal extubation. Respir Care. 2014;59(6):991-1002.

12. Ferrario L. Extubation catheters. In: Hagberg CA, Artime CA, Daily WH, editors. The difficult airway: a practical guide. Oxford: Oxford University Press; 2013:136.

13. Brull SJ, Kopman AF. Current status of neuromuscular reversal and monitoring: challenges and opportunities. Anesthesiology. 2017;126(1):173-90.

14. Ochoa ME, Marín Mdel C, Frutos-Vivar F, et al. Cuff-leak test for the diagnosis of upper airway obstruction in adults: a systematic review and meta-analysis. Intensive Care Med. 2009;35(7):1171.

15. Girard TD, Alhazzani W, Kress JP, et al. ATS/CHEST Ad Hoc Committee on Liberation from Mechanical Ventilation in Adults. An Official American Thoracic Society/American College of Chest Physicians Clinical Practice Guideline: liberation from mechanical ventilation in critically ill adults. Rehabilitation protocols, ventilator liberation protocols, and cuff leak tests. Am J Respir Crit Care Med. 2017;195(1):120-33 Recent clinical practice guidelines on liberation from mechanical ventilation in critically ill patients.

16. Popat M, Mitchell V, Dravid R, Patel A, Swampillai C, Higgs A. Difficult Airway Society Guidelines for the management of tracheal extubation. Anaesthesia. 2012;67:318-40 Guidelines on extubation. Helpful algorithms included.

17. Tung A, Fergusson NA, Ng N, Hu V, Dormuth C, Griesdale DEG. Medications to reduce emergence coughing after general anaesthesia with tracheal intubation: a systematic review and network metaanalysis [published online ahead of print, $2020 \mathrm{Feb} 22$ ]. Br J Anaesth. 2020;S0007-0912(20)30012-X. https://doi.org/10.1016/j. bja.2019.12.041

18. Apfelbaum JL, Hagberg CA, Caplan RA, et al. American Society of Anesthesiologists Task Force on Management of the Difficult Airway. Practice guidelines for management of the difficult airway: an updated report by the American Society of Anesthesiologists Task Force on management of the difficult airway. Anesthesiology. 2013;118(2):251.

19. Law JA, Broemling N, Cooper RM, et al. Canadian Airway Focus Group. The difficult airway with recommendations for management-part 1-difficult tracheal intubation encountered in an unconscious/induced patient. Can J Anaesth. 2013;60(11):1089 118 . 
20. Law JA, Broemling N, Cooper RM, et al. Canadian Airway Focus Group. The difficult airway with recommendations for management-part 2-the anticipated difficult airway. Can J Anaesth. 2013;60(11):1119-38.

21. Mort TC, Braffett BH. Conventional versus video laryngoscopy for tracheal tube exchange: glottic visualization, success rates, complications, and rescue alternatives in the high-risk difficult airway patient. Anesth Analg. 2015;121(2):440-8.

22. Cooper RM, O'Sullivan E, Popat M, Behringer E, Hagberg CA. Difficult Airway Society guidelines for the management of tracheal extubation. Anaesthesia. 2013;68(2):217.

23. Cooper RM, McCarthy S, Urdaneta F. Tolerability of the Cook staged extubation wire. Anaesthesia. 2018;73(9):1169-70.

24. Duggan LV, Law JA, Murphy MF. Brief review: Supplementing oxygen through an airway exchange catheter: efficacy, complications, and recommendations. Can J Anaesth. 2011;58(6):560 Excellent review on the safe use of airway exchange catheters.

25. Nair I, Bailey PM. Use of the laryngeal mask for airway maintenance following tracheal extubation. Anaesthesia. 1995;50(2):174 5 .
26. Esteban A, Frutos-Vivar F, Ferguson ND, Arabi Y, Apezteguía C, González M, et al. Noninvasive positive-pressure ventilation for respiratory failure after extubation. N Engl J Med. 2004;350(24): 2452-60.

27. Hernández G, Vaquero C, Colinas L, Cuena R, González P, Canabal A, et al. Effect of postextubation high-flow nasal cannula vs noninvasive ventilation on reintubation and postextubation respiratory failure in high-risk patients: a randomized clinical trial. JAMA. 2016;316(15):1565-74.

28. Ouellette DR, Patel S, Girard TD, Morris PE, Schmidt GA, Truwit JD, et al. Liberation from mechanical ventilation in critically ill adults: an official American College of Chest Physicians/ American Thoracic Society Clinical Practice Guideline: inspiratory pressure augmentation during spontaneous breathing trials, protocols minimizing sedation, and noninvasive ventilation immediately after extubation. Chest. 2017;151(1):166-80.

Publisher's Note Springer Nature remains neutral with regard to jurisdictional claims in published maps and institutional affiliations. 